\title{
HDR10+ Concepts, Principles, Capabilities and Advantages for the Next-Generation of Brazilian Broadcasting System (TV 3.0)
}

\author{
Rodrigo Admir Vaz \\ Luiz Gustavo Pacola Alves \\ Steve Larson
}

CITE THIS ARTICLE

Vaz, Rodrigo Admir; Alves, Luiz Gustavo Pacola; Larson, Steve; 2021. HDR10+ Concepts, Principles, Capabilities and Advantages for the Next-Generation of Brazilian Broadcasting System (TV 3.0). SET INTERNATIONAL JOURNAL OF BROADCAST ENGINEERING. ISSN Print: $2446-9246$ ISSN Online: 2446-9432. doi: 10.18580/setijbe.2021.2. Web Link: http://dx.doi.org/10.18580/setijbe.2021.2 


\title{
HDR10+ Concepts, Principles, Capabilities and Advantages for the Next-Generation of Brazilian Broadcasting System (TV 3.0)
}

\author{
Vaz, Rodrigo Admir; Alves, Luiz Gustavo Pacola; Larson, Steve \\ SIDIA Instituto de Ciência e Tecnologia - DTV Lab. \\ Samsung - SRA
}

\begin{abstract}
This paper aims to briefly introduce the current Brazilian DTV scenario towards the next generation DTT system (TV 3.0) as well as HDR (High Dynamic Range) principles, features and applications. Different HDR formats have been proposed along the history with different features and purposes. One of them is the HDR10+ technology that can provide powerful advantages and benefits related to picture quality and based on dynamic metadata system. HDR10+ is being adopted by major international digital terrestrial TV systems and other standardizations bodies. In this scenario TV 3.0 can take advantage of HDR10+ to improve the overall system from industry perspective, and consequently delivering a consistent user experience.
\end{abstract}

Key Words - HDR, HDR10+, TV 3.0, Video Quality, Nextgeneration of broadcasting standards and systems, Video coding and processing

\section{INTRODUCTION}

Tn the second half of 2020, SBTVD (Sistema Brasileiro de 1 Televisão Digital - Brazilian Digital Television System) Forum - association responsible to the make DTV (digital terrestrial television) system specifications used in Brazil released CfP (Call for Proposals) with the requirements of the next Brazilian terrestrial DTV system (named TV 3.0), open to all international associations to deliver respective proposals as solutions to the requirements. TV 3.0 CfP requirements were divided in groups, as physical and transport layers, audio and video coding, captions, and application coding.

During the first half of 2021, SBTVD Forum defined the test procedures to evaluate the candidate solutions, and during the second half 2021, SBTVD Forum Test Labs will be testing proposed solutions [1].

Several features are part of the requirements of TV 3.0, as $\mathrm{C} / \mathrm{N} \leq 0 \mathrm{~dB}, \mathrm{MIMO}$ (Multiply Input Multiple Output) 2x2 support, channel bonding, vital Broadband and Broadcast integration, emergency warnings, accessibility, immersive audio, scalable audio, and video. Especially for video, UHD (Ultra-High Definition) 4K and 8K image, HFR (High Frame Rate), WCG (Wide Color Gamut) and HDR (High Dynamic

\footnotetext{
Vaz, Rodrigo Admir, studies Ph.D. in digital TV at Mackenzie Presbyterian University, besides being a senior engineer at SIDIA DTV Lab (e-mail: rodrigo.vaz@samsung.com).

Alves, Luiz Gustavo Pacola is MSc by University of São Paulo and technical coordinator at SIDIA DTV Lab (e-mail: luiz.alves@samsung.com).

Larson, Steve is project manager at Samsung SRA US Lab (e-mail: steve.1@samsung.com).
}

Range) support are required.

Varied solutions were proposed by the consortiums to meet TV 3.0 requirements, as MMTP (MPEG Multimedia Transport Protocol), ROUTE (Real-time Object delivery over Unidirectional Transport) for transport layer; MPEG-H, AVSA and AC-4 for audio coding; H.266, H.265 and AVSA for video coding; SMPTE ST 2094-10 (Dolby Vision), SMPTE ST 2094-20, SMPTE ST 2094-30 (SL-HDR (1/2/3)) and SMPTE ST 2094-40 (HDR10+) for HDR Dynamic Mapping Codec [1].

HDR is a technology used to improve picture quality, better representing luminance, and colors in videos images. It is contrasted with SDR (standard dynamic range), which has become the term for older technology. HDR offers the possibility to represent more realistic images with substantially brighter highlights, darker shadows, and more colors than what was previously possible. HDR enables better use of displays that have high brightness, contrast, and color capabilities.

A HDR technology encompasses various technical characteristics, such as transfer function, dynamic or static metadata, bit depth, maximum luminance range, and color volume. Some HDR formats have been proposed since 2014 and the most common formats are Dolby Vision, HLG, SLHDR, HDR10 and HDR10+ [2].

HDR10+ has been established as SMPTE standard ST 2094-40 [3], and is a video codec agnostic solution which aligned with TV 3.0 video requirements [1] can leverage next generation TV experiences and bring several advantages to the new Brazilian DTV system: enhanced video quality, better user experience on consumer TVs.

Dynamic tone mapping is another important characteristic offered by HDR10+. Such feature applies a different tone mapping curve from scene to scene, maximizing image quality while preserving the creative intent. Dynamic tone mapping eliminates clipping in highlights and crushing in the darks [3].

As a royalty free technology with a simple production workflow, the adoption and device expansion have experienced significant growth since HDR10+'s launch, allowing worldwide consumers to access the new technology. An ever-expanding catalog of international video content is already produced with HDR10+ and also available through major content and streaming video providers. Professional tool development is facilitated with a number of commercial and royalty free SW development kits and cloud CLI 
(command-line interface) tools available for implementing HDR10+ into almost any application.

This paper describes several advantages offered by HDR10+ technology to improve TV 3.0 system and offer enhanced experience to the end user and is organized as follows. Section II provides an introduction to fundamentals of image quality and importance of HDR. Section III shows HDR working principle. Section IV illustrates the workflows to produce HDR10+ video contents. Section V shows HDR10+ in practice. Section VI details benefits, and advantages offered by HDR10+ paving the way to TV 3.0 and Section VII concludes this paper.

\section{IMAGE QUALITY FUNDAMENTALS AND HDR IMPORTANCE}

Display devices such as LCD (Liquid Crystal Displays) with global backlight screens, OLEDs (Organic LightEmitting Diodes), QD (Quantum Dot) enhancement layers, direct-view LED technology and others are acquiring the ability to show an increased range of brights, darks, and color, improving image quality, leading to an increased realism [4].

\section{A. Image Quality Elements}

Considering this image quality improvement, it is possible to highlight five elements, which have a huge influence over image quality [2], [4], [5].

- Spatial Resolution: Bigger spatial resolution shows better image details and increases the image perceived depth sensation. Currently, the highest video resolutions used are $4 \mathrm{~K}(3840 \times 2160)$ and $8 \mathrm{~K}(7680 \times 4320)$ UHD (Ultra high Definition).

- Frame Rate (also known as temporal resolution): Higher frame rate is especially beneficial for scenes with significant motion, such as sporting events, or action movies since it decreases the image blur. Most modern video content are produced using 60 or $120 \mathrm{fps}$.

- Wide Color Gamut: Modern HDR displays are capable of showing a wider color spectrum than ever before. Current displays accept an input signal with a BT 2020 [6] color gamut.

- Color Depth (also known as Bit Depth): Is the quantity of bits used by each color component to compose a pixel. The larger the number of bits used, the smoother the color transition inside the image with be. UHD content supports at least 10 bits per color.

- Luminance: With higher luminance capabilities now available, more realistic luminance variation between scenes such as sunlight, indoor, and night scenes can be shown, as well higher contrasts can be explored. Presently, luminance limits used in the video content converted from SDR (maximum of 100 nits) to HDR limits can reach up to 10,000 nits.

Figure 1 illustrates possible combinations of these elements.

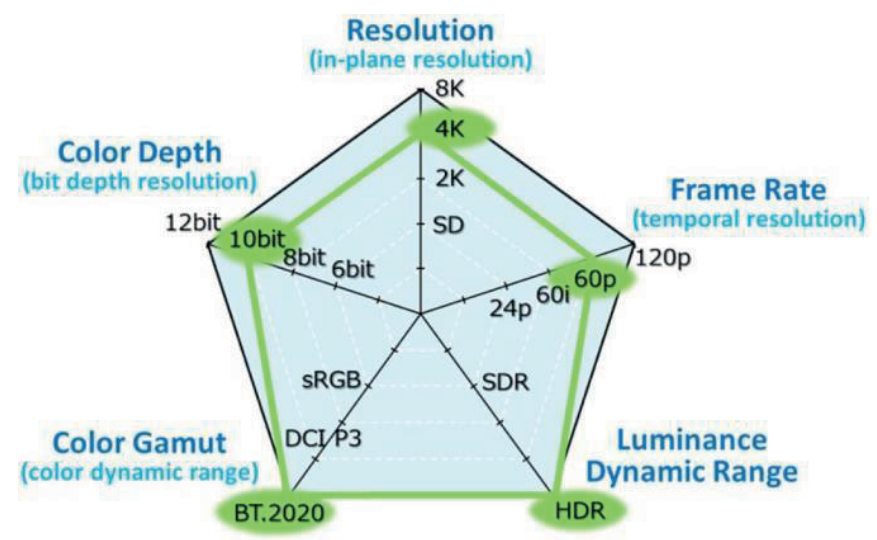

Figure 1- Five Elements of Image Quality Combined - Taken from Reference [2].

\section{B. HDR Importance}

All these elements combined leads to image quality improvement, allowing the display device to show more realist images, with greater picture detail, more vibrant colors, and smoother motion.

HDR has a great impact on image quality, delivering darker "darks" and brighter "brights" along with more nuanced gradations for better delineation of on-screen shapes. This feature offers some benefits as below [4].

- Perceptual Benefits: The human eye is capable of sensing light values from starlight to bright sunlight, a $10^{14}$ range of illuminance. However, total dynamic range is only achieved after many minutes of night vision adaptation, at any given moment, only a fraction of that range is available: estimated at around 13 to 16 stops (about $10^{5}$ dynamic range).

Using SDR, the original TV imaging system could only capture, record, transmit and display less than 10 stops of dynamic range, limiting how content creators could convey scene brightness. This problem persisted with the launch of HDTV in the 1990's.

HDR takes advantage of the latest cameras, processing, storage, distribution platforms and displays, to maintain as much of the full range of human vision as possible from the original scene all the way through to the display.

- Technical Benefits: HDR technology is often associated with "brighter" pictures measured in "nits". While brightness is a major aspect of HDR - it is only one part of the story. HDR is really about the entire dark-to-light range of tonal values, what cinematographers call "grayscale." HDR enables us to see the entire grayscale: not just the highlights, but all the shadows and all the subtle gradations in between, as depicted in Figure 2.

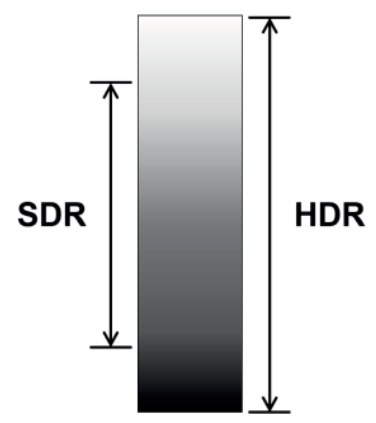

Figure 2 - Today's Cameras Capture Deep Shadows and Bright Highlights. SDR Cannot Reproduce Them. HDR Can. - Taken from Reference [4]. 
- Creative Benefits: Grayscale improvements go directly to the heart of visual storytelling, enabling a greater range of expression. Careful control of grayscale's light and shadow helps to establish mood, convey realistic skin tones, and identify what to look for in a scene. In fact, it is so important that for each individual scene, movie crews typically set up a unique configuration of lights, reflectors, and diffusers to achieve just the right effect.

Moreover, the director and cinematographer continue to perfect grayscale values during postproduction, via color correction and mastering. By expanding and refining the grayscale, HDR dramatically improves movies, TV programs and videogames, making it more engaging, dynamic, and closer to the creative intent.

\section{HDR WORKING PRINCIPLE}

HDR is a technology used to improve picture quality, better representing luminance, and colors in videos images, offering the possibility to represent more realistic images with substantially brighter highlights, darker shadows, and more colorful colors than what was previously possible. This feature enables better use of displays that have high brightness, contrast, and color capabilities.

To take advantage of the increase in the peak luminance, different transfer functions were proposed, namely the PQ (Perceptual Quantizer) curve [7], [8]. This curve is capable of representing luminance level up to 10,000 nits and down to 0.0001 nits.

A transfer function, also known as tone mapping curve, renders incoming HDR contents on a display having a smaller dynamic range, improving image contrast, therefore showing more realistic pictures.

Figure 3 shows the need for HDR technology presenting different video contents.

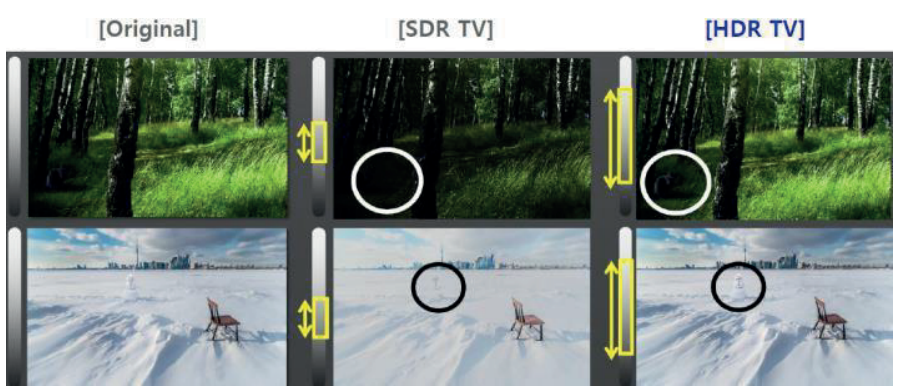

Figure 3 - Difference Among Original, SDR and HDR Contents.

\section{A. Static Tone Mapping}

Early HDR Technologies use static tone mapping, applying one fixed tone mapping curve (transfer function) to all frames of the content. One example of this feature is HDR10 [8], [9], which is a widespread technology, that uses PQ [6], [7] curve and static metadata.

PQ curve enables content creators to exactly specify the image color and brightness as viewed on a reference monitor in the grading suite. It is up to displays to play this directly or adapt to the consumer's viewing environment.

Static metadata includes information about the display on which the content was mastered, information such as Maximum Frame Light Level (MaxFALL) and Maximum Content Light Level (MaxCLL).

HDR10 contents production is shown in Figure 4. Such information is used by the receiving display, to adjust its own brightness for the content, as depicted in Figure 5. The horizontal axis represents complete range of possible light levels in PQ-encoded content. The vertical axis is the light level produced by an HDR10 display.

However, these values remain static throughout the runtime for HDR10 content, which produces less than ideal results wherein some scenes are not as bright as they can be while others could be brighter than they needed to be. This is not efficient approach since dark scene gets dimmer if tone mapping is designed to avoid highlight saturation and highlight gets saturated (banding) if designed to avoid dimming in dark area.

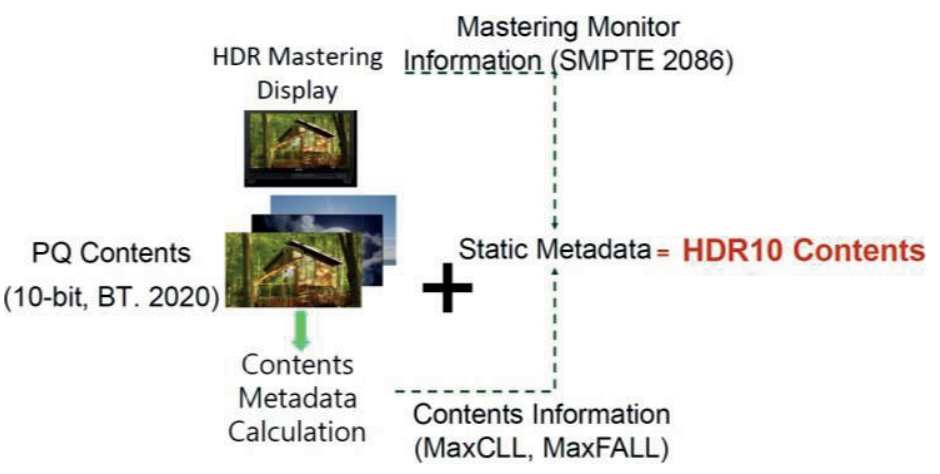

Figure 4 - HDR10 Contents Production.

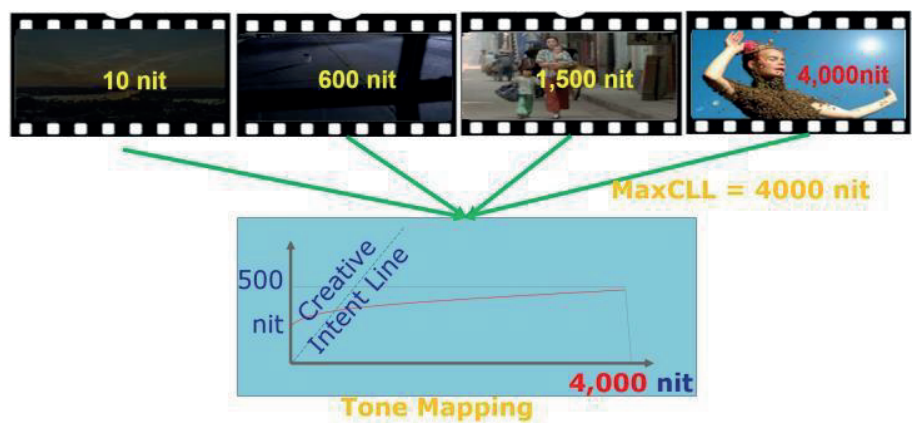

Figure 5 - Static Tone Mapping Applies One Fixed TM Curve to Entire Contents.

\section{B. Dynamic Tone Mapping}

More modern HDR Technologies use dynamic tone mapping, applying scene specific tone curves via dynamic metadata.

With the support of dynamic metadata, information is created for the content on a scene-by-scene or frame-byframe basis for adjusting the content light level. This information can then be used by receiving display to adjust luminance to match the mastered content exactly,reproducing the creator's intent more closely. It is efficient tokeep the creative intent: no excessive dimming, maintained saturation and highlight detail.

An illustration of scene based dynamic tone mapping is depicted in Figure 6. It uses 500 nits as the display peak luminance. Dynamic tone mapping happens when the source content peak luminance is higher than the display device peak luminance.

HDR10+ is a HDR technology, which adds dynamic metadata on top of already existing static HDR10 technology, and is standardized in SMPTE ST 2094-40 [3] spec.

ST-2094-40 metadata includes percentile information calculated on the actual scene content, a guided Opto-optical Transfer Function (OOTF) curve. It allows precise adaptation 
of tone mapping based on Bezier curve $\left(\mathrm{N}^{\text {th }}\right.$ order Bernstein polynomial) to various display capabilities.

Figure 7 shows tone mapping function based on $\mathrm{N}^{\text {th }}$ Bezier curve. The tone mapping function is composed of two sections: The first section is linear while the second section is a polynomial. The two sections are conjoined at a knee point $(\mathrm{Kx}, \mathrm{Ky})$. The coefficients of the Bernstein polynomial along with the coordinates of the knee point are part of the dynamic metadata that defines the scene-specific tone mapping curve [3], [10].

The Figure 8 and Figure 9 show image quality difference between Static and Dynamic tone mapping.

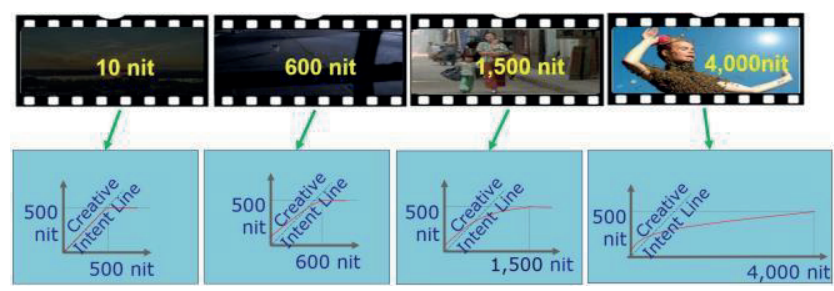

Figure 6 - Dynamic Tone Mapping: Scene Based Tone Curves.

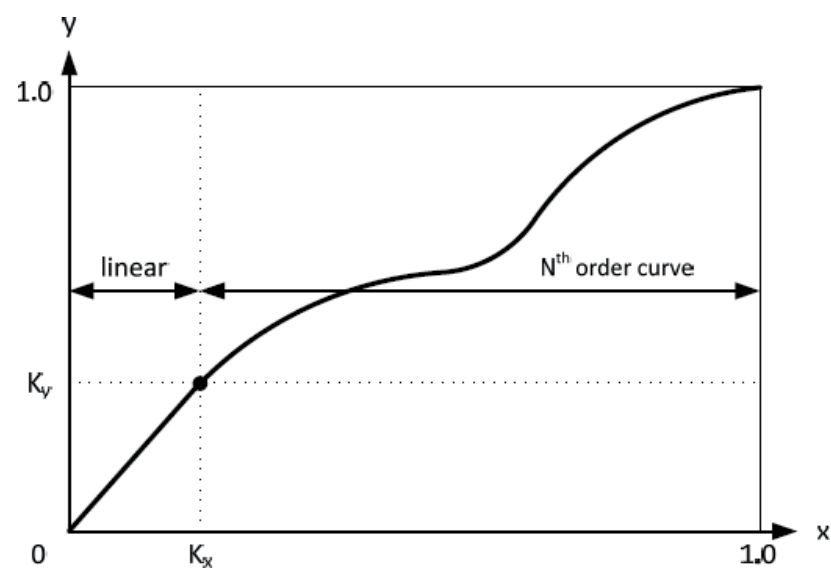

Figure 7 - Tone Mapping Function Based on $\mathrm{N}^{\text {th }}$ Bezier Curve. Taken from Reference [10]

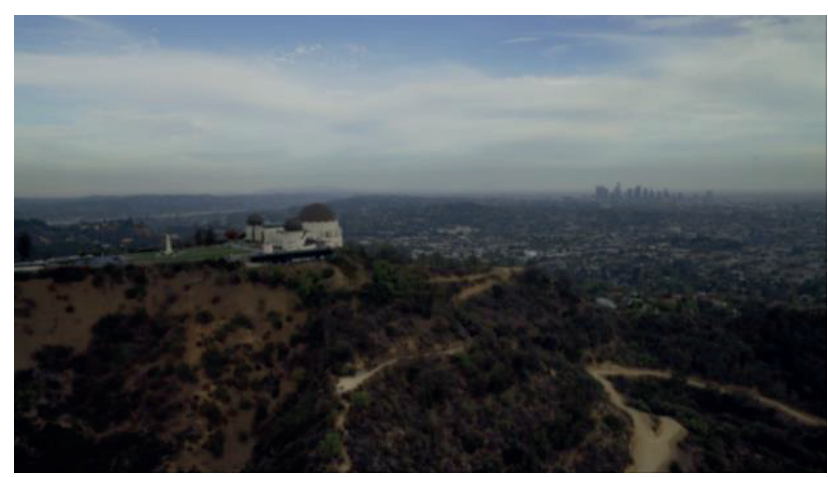

Figure 8 - Static Tone Mapping Image.

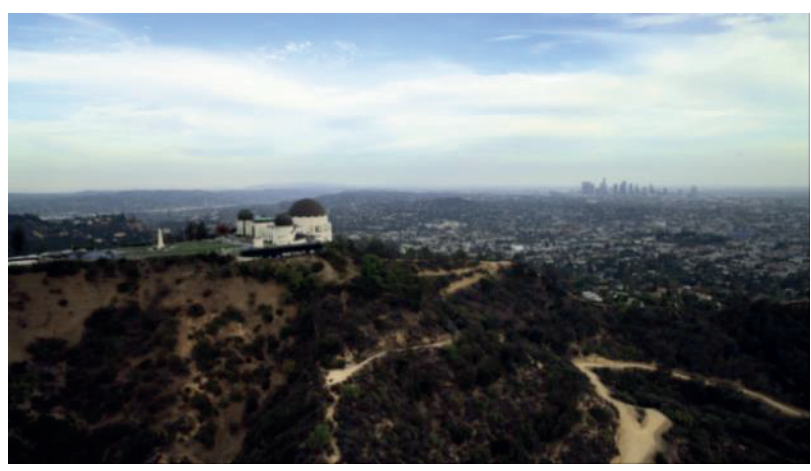

\section{HDR10+ WORKFLOWS}

The HDR10+ workflow is designed to have minimal impact on existing HDR capture, encode, distribution and display infrastructure. HDR10+ dynamic metadata is generated on captured or mastered HDR content and stored in the video stream during or after encoding through low complexity SEI (Supplemental Enhancement Information) messages and is backwards compatible with HDR10 capable devices.

HDR10+ is also supported in several commercial hardware and software encoders for use in both live and off-line workflows. This flexibility allows content owners to update their catalogs easily and rapidly to the latest dynamic metadata standard and take full advantage of dynamic metadata in their live broadcasts.

Tone mapping is applied frame by frame according to video content features and display capabilities allowing it to be fully capable of portraying optimized visuals with consistent color saturation and detail improving user's experience.

These concepts are detailed in the following topics.

\section{A. Live Workflow}

HDR10+ live encoding is easily executed by encoders, which follows workflow shown in Figure 10.

Real time broadcast operations are supported at the point of transmission enabling the flexibility to transmit the video stream either with no metadata or with dynamic metadata. In the second case, metadata is generated and inserted directly into the video stream in parallel with video encoding adding no additional latency to the overall system

The HDR10+ capable encoder will calculate the statistics of the input frame, create the tone curve for the current one, injects into the encoded frame and it can be output as an ES (elementary stream). Such ES is transmitted over terrestrial networks, as shown in Figure 11, and the embedded HDR10+ dynamic metadata is extracted and interpreted in a compatible display to provide scene-by-scene tone mapping to the HDR signal.

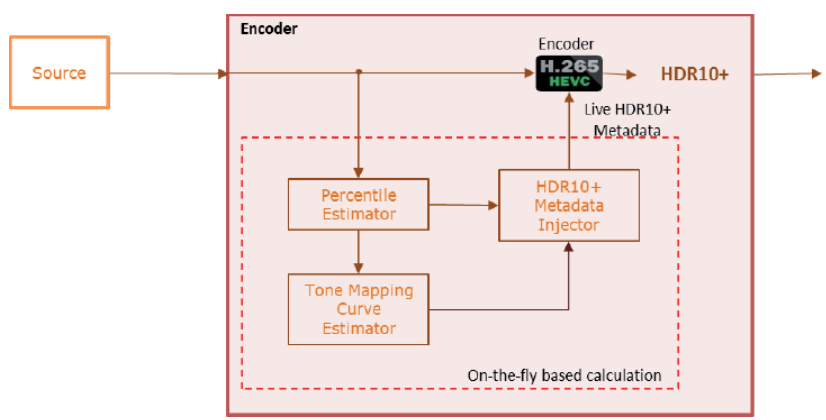

Figure 10 - HDR10+ Encoder for Live Broadcast Environment. Taken from Reference [4].

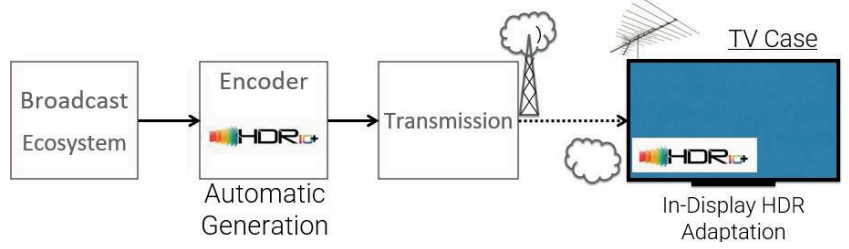

Figure 11 - HDR10+ Live Broadcast Workflow.

Figure 9 - Dynamic Tone Mapping Image. 


\section{B. Off-line Workflow}

In an off-line workflow, HDR10+ metadata is generated on HDR mastered content. For UHD Blu-ray discs, the HDR10+ metadata is inserted in the encoded file during disc authoring. When inserted in an HDR10+ capable UHD Blu-ray player, the metadata is then extracted from the encoded stream and transmitted through the HDMI channel with Vendor Specific Info-frames to the HDR10+ capable display, where the dynamic tone mapping is then applied to the input signal, as depicted Figure 12.

The same process is followed for streaming content or if the TV set receives HDR10+ content directly. The TV detects the presence of HDR10+ metadata in the SEI message of the input stream and applies the tone mapping automatically.

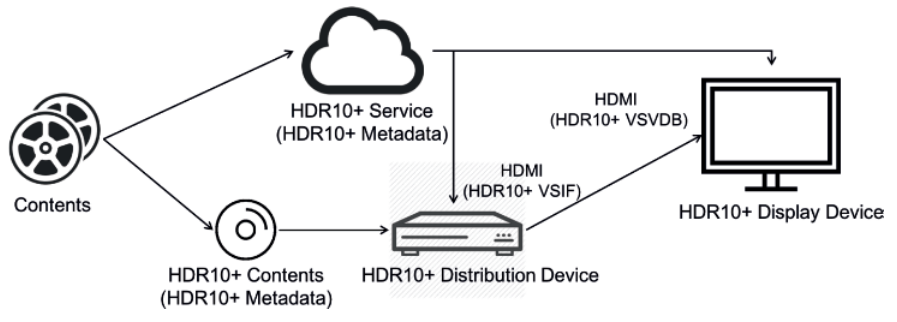

Figure 12 - HDR10+ Off-line Workflow. Taken from Reference [4].

\section{Ecosystem Deployment}

Furthermore, HDR10+ is an open-source, royalty free technology allowing it to be easily adopted and deployed across all devices in an HDR10 ecosystem with very low overhead and cost [4].

The HDR10+ ecosystem is rapidly growing with continued support from major device manufacturers and content creators. Since the creation of the HDR10+ Display Certification Program in 2018 until today, a dozen global display manufacturers have already produced over 4,700 different models of HDR10+ certified displays available to consumers worldwide (more details in section V).

Along with displays, there are also over 140 certified mobile devices, both tablets and smart phones, that support HDR10+ playback and in some cases HDR10+ capture as well allowing for rapid proliferation of HDR10+ content generated by amateurs and enthusiasts.

Professional/theatrical content is abundant and readily available from 5 major Hollywood studios on both UHD Bluray discs and through streaming OTT (Over-the-top) services with thousands of hours of episodic and theatrical pieces utilizing HDR10+ dynamic metadata for consumers to enjoy.

\section{HDR10+/HDR10 Backwards Compatibility}

HDR systems have been deployed since 2015 [2], when studios mastered video contents using PQ curve. HDR10 spec arose as one of the firsts systems, which use this transfer function and static metadata to improve image quality. ST2094-40 dynamic tone-mapping technology is advanced from HDR10, adding dynamic metadata for the same purpose. Such evolution concept is synthetized in the Figure 13.

As part of the HDR10 ecosystem, HDR10+ content works seamlessly with both HDR10 and HDR10+ devices. This content will display on HDR10 devices, which simply ignore the dynamic metadata while HDR10+ capable devices can take advantage of the dynamic metadata. HDR10 contents are totally supported by HDR10+ devices, as Figure 14.

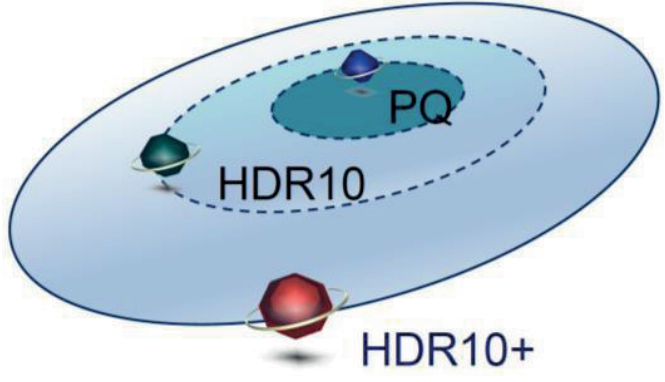

Figure 13 - HDR evolution from PQ to HDR10+.

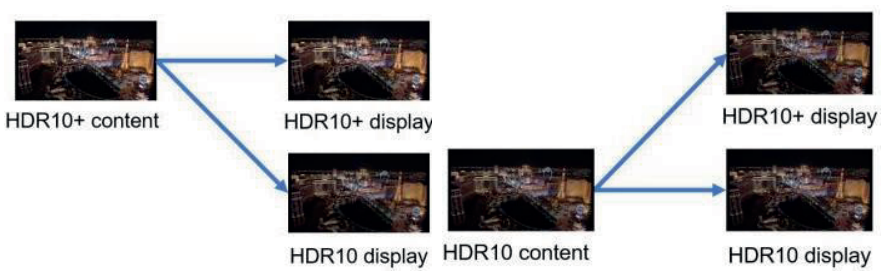

Figure 14 - HDR10+/HDR10 Backwards Compatibility- Taken from Reference [4].

\section{E. Device Tone Mapping}

HDR10+ displays will process the video, frame by frame, with HDR10+ metadata to apply the best tone mapping for the content on the device, as the diagram of Figure 15.

Tone mapping happens when the source content peak luminance is higher than the display device peak luminance. Once HDR10+ content is delivered to a display device, the decoder will parse the HDR10+ metadata and video essence and process through the video pipeline. If a display doesn't support the HDR10+ metadata, it will be simply ignored as any other optional ITU-T T.35 metadata and the display reverts to show the strict version of HDR10.

The existing HDR technology, HDR10, leads to inconsistent reproduction of HDR content from one display device to another as only limited static metadata for content can be provided.

HDR10+ provides articulated scene based statistical data and optional guided tone mapping information to the display. This data enables a consistent reproduction of the source master content across displays of varying capability. Additionally, when a knee point is included with HDR10+ metadata, displays maintain the content's original look in shadow detail as no tone mapping happens below the kneepoint [4].

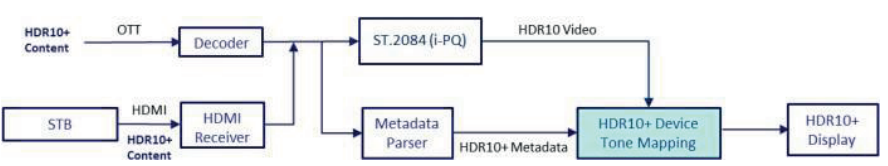

Figure 15 - HDR10+ Device Tone Mapping. Taken from Reference [4]

\section{HDR $10+$ IN PRACTICE}

This section is composed by three different sub-sections. First, introduces certification program promoted by HDR10+ Technologies LLC. Second, presents the standardization efforts by international SDOs (Standards Developing Organizations). The last sub-section brings the results of subjective evaluation tests made by a third-party lab. 


\section{A. Certification Program Available}

In order to promote HDR10+ technology, HDR10+ Technologies LLC [4] was created. The entity administers the license and certification program for products that want to adopt the technology, and provides the technical and test specifications, and certified logo, shown in Figure 16.

Royalty-free SW development kits and cloud CLI tools available for implementing this solution.

Nowadays, on the content production and distribution side, several studios, content distributors and equipments vendor adopted the technology. A current list of HDR10+ adopters can be found on the HDR10+ website, https://hdr10plus.org/adopters/.

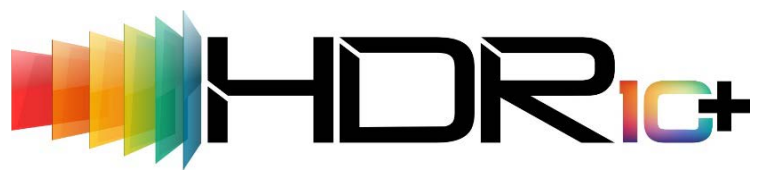

Figure 16 - HDR10+ Logo. Taken from Reference [4].

\section{B. Standardization}

Besides SMPTE 2094-40 spec, HDR10+ technology, from metadata generation to decoding, is fully standardized through multiple SDOs, adopted by DVB and ATSC 3.0 DTV systems, being available to be included in any broadcasting plan as below.

- SMPTE ST 2094-40:2020 [3] - describes how the technology works.

- CTA-861-H (2020) [12] - describes how digital audio/video signals can be sent from one device to another.

- CTA-5001-C (2020) [12] - deals with encoding and packaging of segmented media for delivery and decoding on end user devices in adaptive multimedia presentations.

- $\quad$ SCTE 215-1-1 2020b (Cable TV) [14] - specifies how HDR10+ is used in cable video services applications.

- DVB Blue Book (version A001r17) [15] - specifies how to use ST 2094-40 in DVB system.

- ATSC 3.0 A/341 [15] - specifies how to use ST 2094-40 in ATSC 3.0 system.

\section{Subjective Evaluation}

Following ITU-BT.500 recommendation [16], a subjective performance evaluation [17] of 2094-40 dynamic tone mapping vs BT.2390 [18] representing tone mapping with static HDR metadata was performed by independent third party entity with the following three goals:

1. 2094-40 dynamic tone mapping and static tone mapping based on BT.2390 adaptation were each compared to the original HDR content on the same professional monitor.

2. The subjective overall quality of dynamic tone mapping using 2094-40 is compared against static tone mapping using BT.2390 adaptation, to verify the quality benefit of dynamic tone mapping on the same professional monitor.
3. The subjective overall quality of dynamic tone mapping using 2094-40 is compared against static tone mapping using BT.2390 adaptation, to verify the quality benefit of dynamic tone mapping on the same consumer monitor.

A total of 34 unique video clips with the following variants were used:

A. Original source content in PQ, mastered at 1000 nits.

B. Original source content in PQ, mastered at 4000 nits.

C. HDR clips with static metadata and a curve applied from BT.2390, tone mapping from a 1000 nit source to 400 nits.

D. HDR clips with dynamic metadata and a curve applied from 2094-40, tone mapping from a 1000 nits source to 400 nits.

E. HDR clips with static metadata and a curve applied from BT.2390, tone mapping from 4000 nits source to 400 nits.

F. HDR clips with dynamic metadata and a curve applied from 2094-40, tone mapping from a 4000 nits source to 400 nits.

Clips share the properties indicated in Table I:

TABLE I

CLIP PROPERTIES.

\begin{tabular}{|c|c|}
\hline Property Value & Property Value \\
\hline Resolution & $3840 \times 2160$ \\
\hline Frame rate & $24000 / 1001 \mathrm{~Hz}$ \\
\hline Chroma sampling & $4: 2: 2$ \\
\hline Color bit depth & 10 -bits per color \\
\hline EOTF & PQ \\
\hline Color model & YcbCr \\
\hline Encoding type & ProRes 4444 HQ \\
\hline Color space & $\begin{array}{c}\text { BT.2020 container } \\
\text { (limited to P3) }\end{array}$ \\
\hline Container & Quicktime \\
\hline Range & Legal \\
\hline
\end{tabular}

Taken from Reference [17].

Three test scenarios were identified in order to effectively evaluate static HDR tone mapping using BT.2390 vs 2094-40 dynamic HDR tone mapping, as below:

- Test Scenario I - Evaluation of HDR tone mapping subjective quality (HDR artistic intent preservation): comparing original vs tone mapped result with two identical reference displays (Sony X300).

- Test Scenario II - Evaluation of HDR tone mapping subjective quality for 4000 nits content on two identical reference displays (Sony X300).

- Test Scenario III - Evaluation of HDR tone mapping subjective quality for 4000 nits content on two identical and limited performance consumer displays (Samsung NU8000).

\section{C.1 Test Scenario I}

Static and dynamic HDR systems are evaluated: both tones mapping a 1000 nits source image to 400 nits.

Viewers judge the visual quality difference between the source reference image $(\mathrm{A})$ and the tone mapped content $(\mathrm{C}$ and D) as follows: 
- $\quad$ Original graded source content vs. (C) BT.2390.

- $\quad$ Original graded source content vs. (D) 2094-40.

Test result aims to identify which (if either of the two) tone mapping methods best preserves the appearance of the original source content.

Video Clips used are identified in Table II:

TABLE II

TEST SCENARIO I SEQUENCE - 1000 NIT SOURCE TONE MAPPED

\begin{tabular}{|c|c|c|}
\multicolumn{3}{|c|}{ TO 400 NITS } \\
\hline Clip \# & Clip Name & Standard \\
\hline 1 & Seattle Waterfront & BT. 2390 \\
\hline 2 & Cakes & BT. 2390 \\
\hline 3 & Rock climbers 1 & $2094-40$ \\
\hline 4 & Seattle Waterfront & Identical - Control Clip \\
\hline 5 & Cactus & $2094-40$ \\
\hline 6 & Boys Face/helmet & $2094-40$ \\
\hline 7 & Rock climbers 1 & BT.2390 \\
\hline 8 & Woman Portrait & $2094-40$ \\
\hline 9 & Cactus & BT.2390 \\
\hline 10 & Cakes & $2094-40$ \\
\hline 11 & Woman Portrait & BT.2390 \\
\hline 12 & Boys Face/helmet & BT.2390 \\
\hline
\end{tabular}

Taken from Reference [17].

- Results

The Table III and Figure 17 present the mean opinion score (MOS) and the 95\% confidence interval (CI) for each clip observers were presented with. The control clip (identical on both displays) was voted highly and significantly higher than the BT.2390 rendition for the Seattle Waterfront clip which indicated the observer's ability to identify the difference. Results for 2094-40 were higher in each clip over BT.2390.

The Figure 18 illustrates the density of observers scoring as a single combined score sheet. Larger and darker circles denote greater frequency of observer selection.

TABLE III

AVERAGE MEAN OPINION SCORE (MOS) RESULTS OF TEST SCENARIO I WITH AVERAGE 95\% CONFIDENCE INTERVAL (CI)

\begin{tabular}{|c|c|c|}
\hline & Average MOS & Average CI \\
\hline BT. 2390 & 47.94 & 9.81 \\
\hline $2094-40$ & 78.67 & 7.18 \\
\hline
\end{tabular}

Taken from Reference [17].

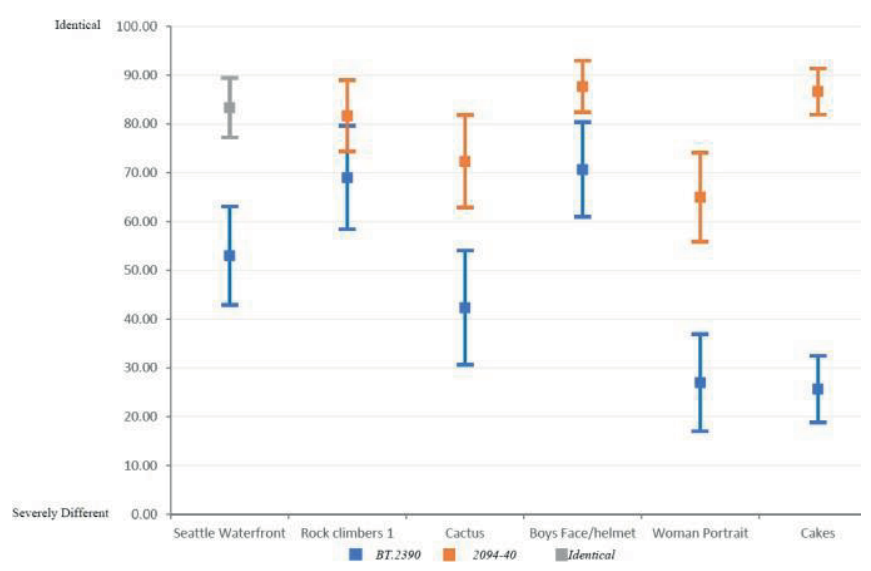

Figure 17 - Mean Opinion Score (MOS) Results of Test Scenario I with 95\% Confidence Interval (CI). Taken from Reference [17]

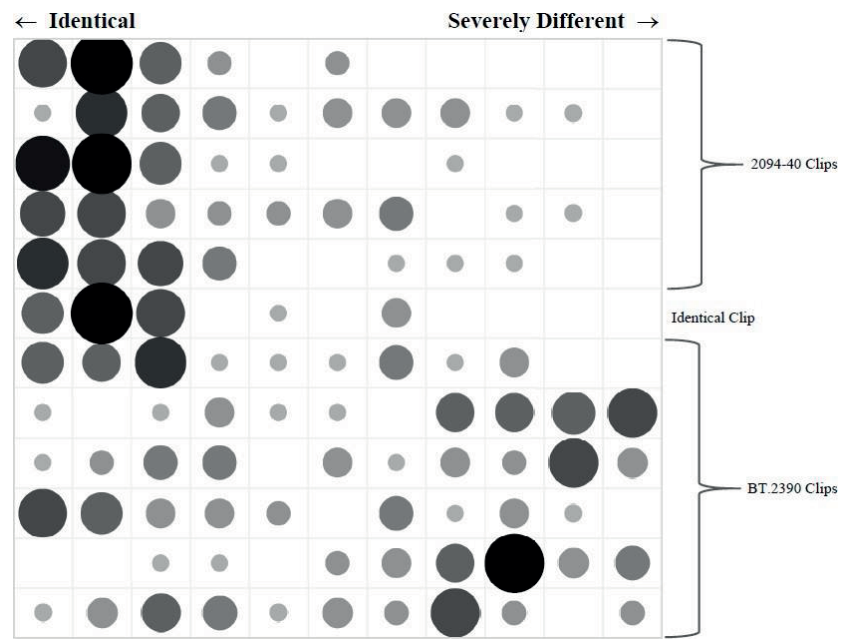

Figure 18 - Mean Opinion Score (MOS) Results of Test scenario I Observer Scoring Sheet. Taken from Reference [17].

\section{C.2 Test Scenario II}

It is compared static vs. dynamic tone mapping of a 4000 nits source image (B) to 400 nits using identical reference monitors and judge which of the two has the better overall visual quality:

- (E) BT.2390 vs (F) 2094-40.

Test result aims to identify which (if either of the two) tone mapping methods has the better subjective overall picture quality.

Video Clips used are identified in Table IV:

TABLE IV

TEST SCENARIO II SEQUENCE - 4000 NIT SOURCE TONE MAPPED TO 400 NITS

\begin{tabular}{|c|c|c|c|}
\hline Clip \# & Clip Name & Left Standard & Right Standard \\
\hline 1 & Airplane takeoff & BT. 2390 & $2094-40$ \\
\hline 2 & Donkeys & BT. 2390 & $2094-40$ \\
\hline 3 & Rock climbers 2 & $2094-40$ & BT.2390 \\
\hline 4 & Alpacas & $2094-40$ & BT.2390 \\
\hline 5 & Water Tower & $2094-40$ & BT.2390 \\
\hline 6 & Canyon Village & BT. 2390 & $2094-40$ \\
\hline 7 & Fire Breather 1 & $2094-40$ & BT.2390 \\
\hline 8 & Swamps & BT. 2390 & $2094-40$ \\
\hline 9 & Inca Ruins & $2094-40$ & BT.2390 \\
\hline 10 & Village Docks & Identical & Identical \\
\hline 11 & Girl Swimming & BT. 2390 & $2094-40$ \\
\hline
\end{tabular}

Taken from Reference [17].

\section{- Results}

The Table V and Figure 19 detail MOS and 95\% CI for each clip observers were presented with. The control clip (identical on both displays) "Village Docks" was clearly identified as the "same" in terms of picture quality in observer voting. The 2094-40 renditions were consistently voted higher than BT.2390. Some 2094-40 renditions were voted significantly higher than their BT.2390 counterparts and others were voted with a smaller improvement.

The Figure 20 illustrates the density of observers scoring as a single combined score sheet, showing a favoring of the 2094-40 renditions along with a clear identification of the control clip.

TABLE V

MOS AVERAGE OF TEST SCENARIO II AND 95\% CI ACROSS ALL CLIPS

\begin{tabular}{|c|c|}
\multicolumn{2}{c}{ CLIPS } \\
\hline Average MOS & Average CI \\
\hline 26.55 & 5.52 \\
\hline
\end{tabular}

Taken from Reference [17]. 


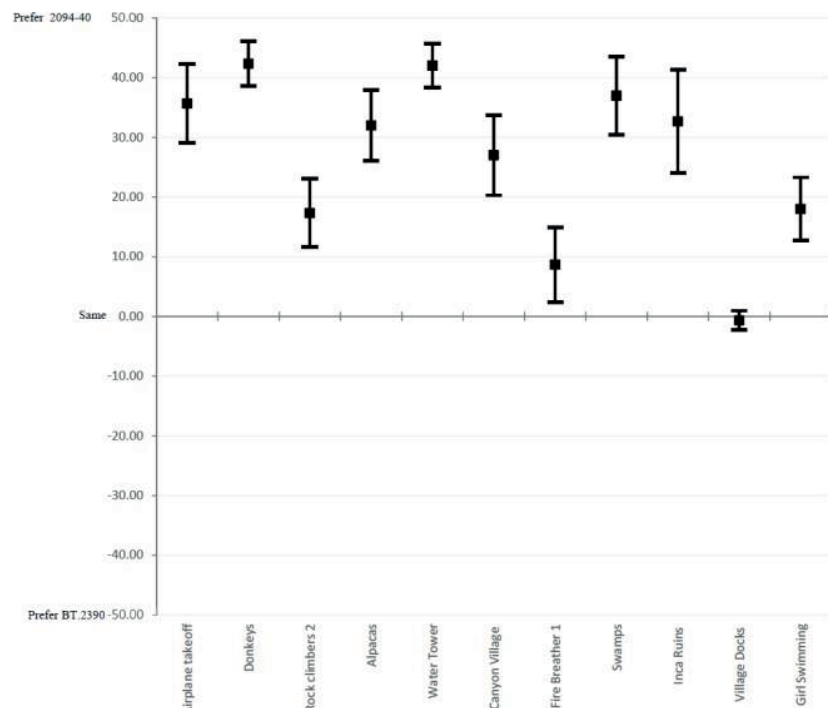

Figure 19 - MOS Results of Test Scenario II with 95\% CI. Taken from Reference [17].

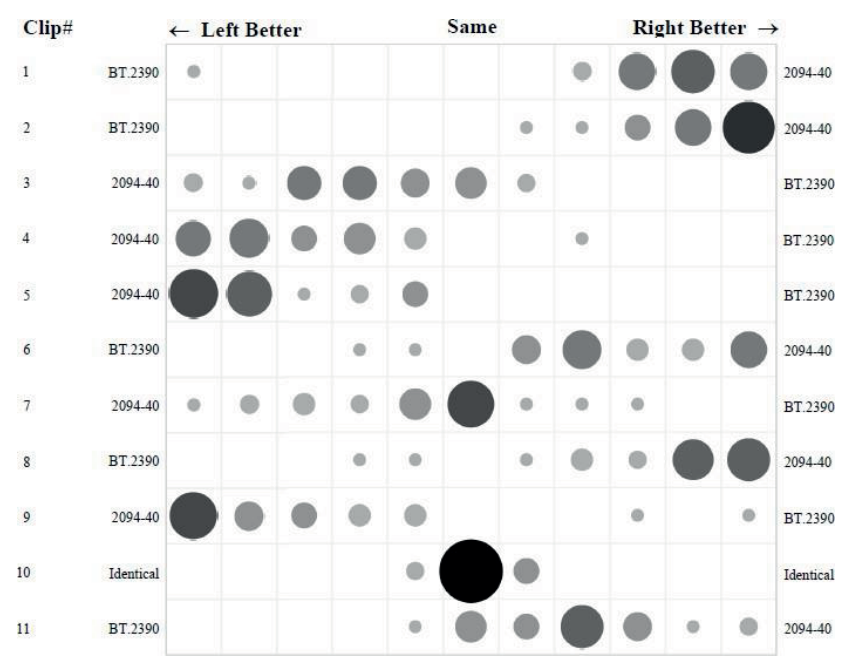

Figure 20 - MOS Results of Test Scenario II - Observer Scoring Sheet. Taken from Reference [17].

\section{C.3 Test Scenario III}

It is compared static vs. dynamic tone mapping of a 4000 nits source image (B) to 400 nits and judge which of the two has the better overall visual quality using limited performance consumer TV:

- (E) BT.2390 vs (F) 2094-40.

Test result aims to identify which (if either of the two) tone mapping methods has the better overall picture quality using limited performance consumer displays

Video Clips used are identified in Table VI:

TABLE VI

TEST SCENARIO III SEQUENCE - 4000 NIT SOURCE TONE MAPPED TO 400 NITS

\begin{tabular}{|c|c|c|c|}
\hline Clip \# & Clip Name & Left Standard & Right Standard \\
\hline 1 & Girl Swimming & $2094-40$ & BT. 2390 \\
\hline 2 & Inca Ruins & BT. 2390 & $2094-40$ \\
\hline 3 & Rock climbers 2 & $2094-40$ & BT. 2390 \\
\hline 4 & Water Tower & $2094-40$ & BT. 2390 \\
\hline 5 & Airplane takeoff & BT. 2390 & $2094-40$ \\
\hline 6 & Canyon Village & $2094-40$ & BT. 2390 \\
\hline 7 & Swamps & BT. 2390 & $2094-40$ \\
\hline 8 & Fire Breather 1 & BT. 2390 & $2094-40$ \\
\hline 9 & Donkeys & BT. 2390 & $2094-40$ \\
\hline 10 & Village Docks & Identical & Identical \\
\hline 11 & Alpacas & $2094-40$ & BT. 2390 \\
\hline
\end{tabular}

Taken from Reference [17].

\section{- Results}

The Figure 21 and Table VII detail the mean MOS and the 95\% CI for each clip observers were presented. Like test scenario II the control clip (identical on both displays) "Village Docks" was clearly identified as the "same" in terms of picture quality in observer voting as well as the 2094-40 renditions being consistently voted higher than BT.2390.

The Figure 22 illustrates the density of observers scoring as a single combined score sheet, showing a favoring of the 2094-40 along with a clear identification of the control clip in the center.

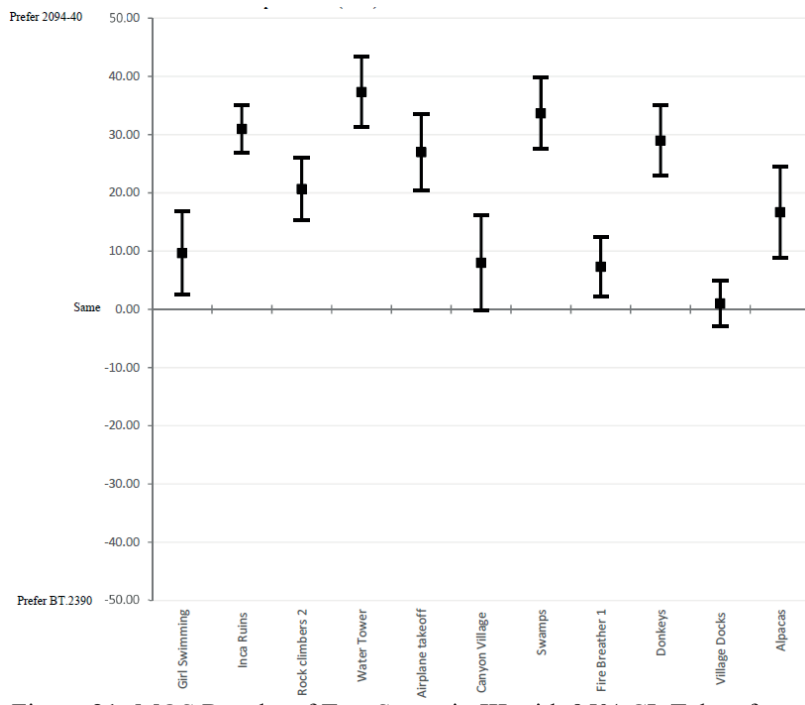

Figure 21- MOS Results of Test Scenario III with 95\% CI. Taken from Reference [17]

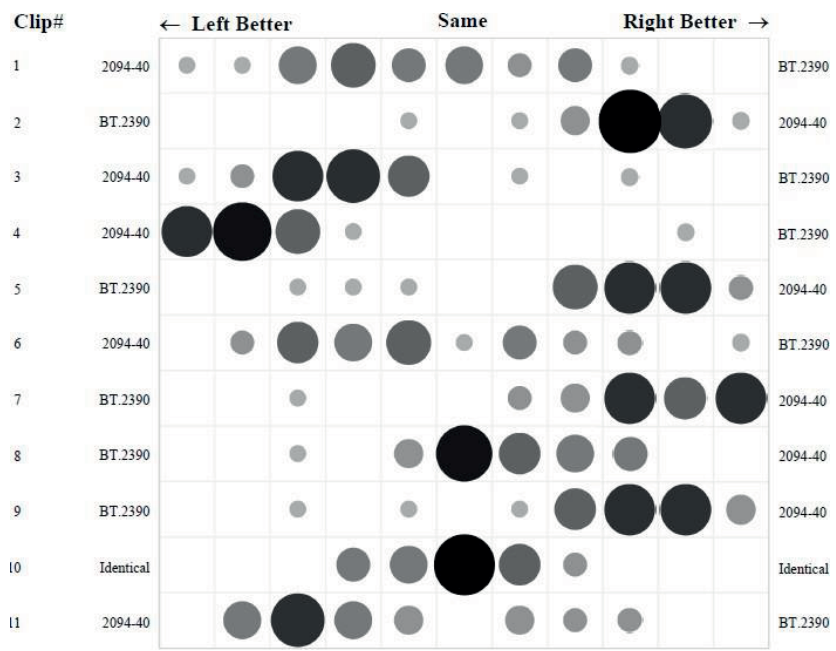

Figure 22- MOS Results of Test Scenario III - Observer Scoring Sheet. Taken from Reference [17].

TABLE VII

MOS AVERAGE OF TEST SCENARIO III AND 95\% CI ACROSS ALL

\begin{tabular}{|c|c|}
\hline Average MOS & Average CI \\
\hline 20.12 & 6.02 \\
\hline \multicolumn{2}{|c|}{ Taken from Reference [17] }
\end{tabular}

\section{C.4 Test Scenario II and III Comparison}

The Figure 23 shows MOS comparison between test scenarios II and III, indicating a clear favoring of 2094-40 HDR format. 


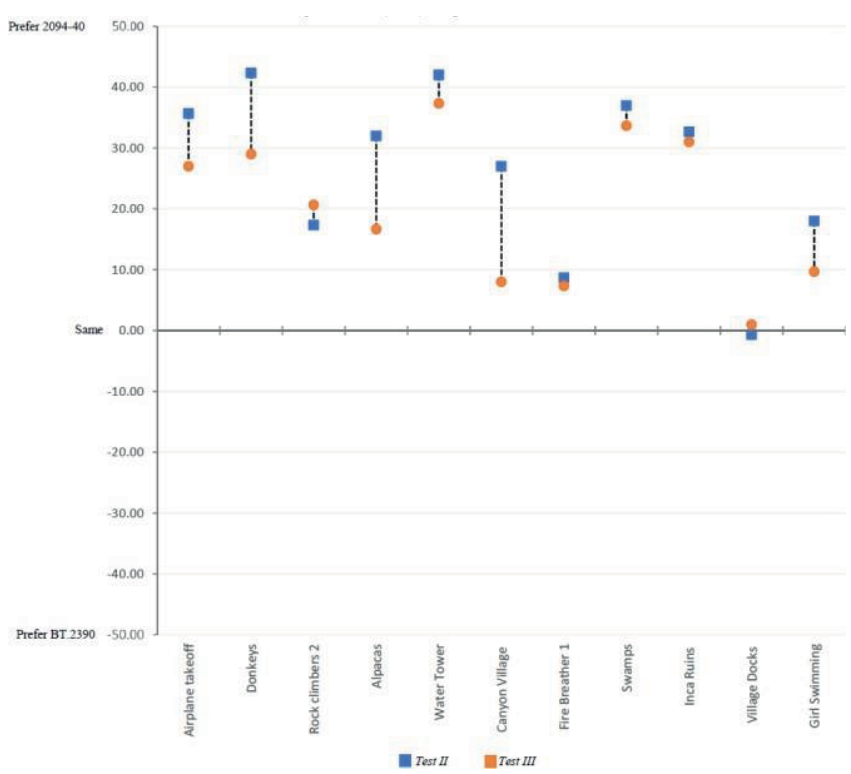

Figure 23 - MOS Comparison of Tests II and III Results. Taken from Reference [17].

\section{HDR 10+ BENEFITS AND ADVANTAGES FOR TV 3.0}

HDR10+ technology enhances image quality, providing a better user experience on consumer receiving displays. It complies with TV 3.0 requirements for video sub-component HDR Dynamic Mapping Codecs, and embraces the following characteristics [1], [3], [4]:

- As resolution agnostic technology, it can support any resolution used in TV $3.0(2 \mathrm{~K} / 4 \mathrm{~K} / 8 \mathrm{~K}$, etc.).

- Able to be supported on any NAL based codec with support already in place for HEVC, VP9 and AV1.

- Bit representation: 10-bit or more (up to 16-bit), more smoothly showing color transition. TV 3.0 requires 10 -bit as bit depth.

- Color space: WCG (ITU-R BT.2100/BT.2020) [6], [7], being able to reproduce more colors.

- 60 fps and lower frame rates demanded in TV 3.0 CfP. The $120 \mathrm{fps}$ support is under development. The higher the frame rate, the smaller the blur presented in action content.

- Pixel representation: 10,000 nits per $\mathrm{R} / \mathrm{G} / \mathrm{B}$, taking full advantage of PQ curve.

- Can be deployed over terrestrial broadcasting systems, as well as in broadband delivery, as expected by TV 3.0. Therefore, improving user's experience in the different ways TV content can be delivered.

- Its dynamic metadata are generated automatically (i.e., without operator intervention) and have been implemented in Live TV and VoD applications, being ready for all TV 3.0 signal transmission possibilities.

Moreover, HD10+ provides powerful advantages like [1] [3], [4]:

- Robust standardization: HDR10+ has been established as SMPTE standard ST 2094-40, broadly standardized by many international associations, and work continues on additional standards. Ratifying HDR10+ Technologies LLC commitment in continuously support HDR10+ technology.

- Well-established deployment across HDR ecosystem streaming platforms, TV manufacturers, mobile device manufacturers, streaming devices, Blu-ray disc players, A/V receivers, SoC makers, professional tools, international video distributors, OTT providers and others, facilitating user's access to this technology.

- Royalty Free: it is open and royalty free solution, facilitating interoperability and broader adoption, which helps industry improve the technology. The only one free of various other dynamic tone-mapping technologies.

- $100 \%$ backward compatible with HDR10, allowing user to enjoy HDR experience even if it has only a HDR10 compatible receiver.

- To deliver all these technical benefits as a consistent user experience, HDR10+ Technologies, LLC was created. Since June 2018, the organization has been certifying HDR10+ compatible content, devices, tools, and services. This also helps promote consumer awareness.

- HDR10+ is an open standard that any manufacturer or content producer can embrace without paying hefty licensing fees.

A comparison among HDR10+ and other HDR technologies are shown in Table VIII:

TABLE VIII

HDR SYSTEMS TECHNICAL FEATURES COMPARISON

\begin{tabular}{|c|c|c|c|c|c|c|}
\hline System & $\begin{array}{l}\text { Transfer } \\
\text { Function }\end{array}$ & Metadata & $\begin{array}{c}\text { Bit } \\
\text { Depth }\end{array}$ & $\begin{array}{l}\text { Color } \\
\text { Gamut } \\
\text { Limit }\end{array}$ & $\begin{array}{l}\text { Max. } \\
\text { Range }\end{array}$ & $\begin{array}{c}\text { Royalty } \\
\text { Paid }\end{array}$ \\
\hline $\begin{array}{c}\text { HLG } \\
\text { (Hybrid } \\
\text { Log } \\
\text { Gamma) }\end{array}$ & $\begin{array}{c}\text { HLG } \\
\text { (BT.2100) }\end{array}$ & No & $\begin{array}{l}\text { 8-bit, } \\
\text { 10-bit }\end{array}$ & BT. 2020 & $100 \%$ & No \\
\hline HDR10 & $\begin{array}{c}\text { PQ } \\
\text { (ST2084/ } \\
\text { BT.2100) }\end{array}$ & $\begin{array}{c}\text { Static } \\
(\text { ST2086) }\end{array}$ & 10-bit & BT. 2020 & $\begin{array}{c}10,000 \\
\text { nits }\end{array}$ & No \\
\hline HDR10+ & $\begin{array}{c}\text { PQ } \\
\text { (ST2084/ } \\
\text { BT.2100) }\end{array}$ & $\begin{array}{c}\text { Static } \\
(\text { ST2086)/ } \\
\text { Dynamic } \\
(\text { ST2094- } \\
40)\end{array}$ & $\begin{array}{l}\text { 10-bit } \\
\text { up to } \\
16 \text {-bit }\end{array}$ & BT. 2020 & $\begin{array}{c}10,000 \\
\text { nits }\end{array}$ & No \\
\hline $\begin{array}{l}\text { Dolby } \\
\text { Vision }\end{array}$ & $\begin{array}{c}\text { PQ } \\
\text { (ST2084/ } \\
\text { BT.2100) }\end{array}$ & $\begin{array}{c}\text { Static } \\
\text { (ST2086)/ } \\
\text { Dynamic } \\
\text { (ST2094- } \\
10)\end{array}$ & $\begin{array}{l}\text { 8-bit } \\
\text { up to } \\
\text { 12-bit }\end{array}$ & BT. 2020 & $\begin{array}{c}10,000 \\
\text { nits }\end{array}$ & Yes \\
\hline SL-HDR & $\begin{array}{c}\text { HLG/PQ } \\
\text { (BT.2100) }\end{array}$ & $\begin{array}{c}\text { Static } \\
\text { (ST2086)/ } \\
\text { Dynamic } \\
\text { (ST2094- } \\
\text { 20/30) }\end{array}$ & $\begin{array}{l}\text { 8-bit, } \\
\text { 10-bit }\end{array}$ & BT. 2020 & $\begin{array}{c}10,000 \\
\text { nits }\end{array}$ & Yes \\
\hline
\end{tabular}

The technology may offer essential benefits to all TV 3.0 ecosystem, as below [1], [3], [4].

\section{A. Public (Consumers)}

- HDR10+ is the most advanced dynamic metadata technology, offering enhanced video quality, allowing improved user experience on consumer displays.

- Subjective evaluation results indicate that HDR content tone mapped using 2094-40 can produce a closer reproduction of the reference image and better perceptible 
overall image quality compared to HDR content tone mapped with BT.2390 [17].

- In the test scenario I results shows a consistently measured opinion that 2094-40 better represented the source image appearance to a lay viewer and is able to more closely maintain a perceptible visual similarity to the original source content than had it been tone mapped with BT.2390 [17].

- This is a royalty free technology, allowing the user to take advantage of its benefits, without additional cost to their equipment. It brings excellent scale representation for brightness and darks. HDR10+ can enable the creative intent to be expressed precisely, resulting in a consistent content reproduction with a more realistic, exciting, captivating entertainment experience.

- Growing industry support. Over 100 companies have already signed on as HDR10+ adopters, and the support from movie studios, streaming companies and list of device manufacturers is growing. Real opportunity for the user to appreciate its favorite content with enhanced video quality.

\section{B. Broadcasters}

- Simple program production. content creators, such as broadcasters, can focus on making the best HDR content, knowing that HDR10+ consumer devices will provide optimum performance. HDR10+ mastering is straightforward and is supported by professional production tools, which also make it simple to upgrade previously produced HDR10 content to HDR10+. In summary, HDR10+ workflow is simple, offering no significant impact to broadcasters.

- Provides better qualified metadata than simply HDR10 and total system compatibility.

- HDR10+ identifies the most important areas of each scene to improve the reproduction of those areas, retaining details in the highlights and shadows across all types of display capabilities.

- Expanding list of HDR10+ content available to consumers from major content providers on both UHD Blu-ray discs and streaming services.

- Allows broadcaster to improve audience experience, offering latest video content with enhanced video quality to a huge number of displays, which supports the technology.

\section{Industry}

- Technology well established in the marketplace, which improves the reliability and longevity of the technology.

- Certification program launched by HDR10+ LLC, which provides further quality assurance, allowing certification logo only for products that pass the rigorous testing requirements leading to playback compliance.

- Adoption based on open technology. Ease of access to anyone who wants to implement the technology. Also, popular professional and open-source tools available for implementing HDR10+ solution (mastering, postproduction, encoding, authoring) on content and devices.
- Ease of implementation \& cost effective for operation. HDR10+ works with a range of video codecs, requires no licensing fees to take full advantage of the brightness of each specific display technology.

- HDR10+ technology is standardized by international standardization bodies recognized by SBTVD Forum [1].

\section{CONCLUSIONS}

Brazil is at the verge of starting the development of the next generation TV Broadcasting system (TV3.0), a disruptive system expected to go 'on the air' in upcoming years. The elaboration of standards is scheduled for 2022 and HDR Dynamic Mapping has been a key-point of TV 3.0, established to improve video technology, adequate to consumer electronics display evolution. Therefore, this paper initiates providing an overview of five elements with huge influence over image quality, HDR importance, working principals, main features and benefits.

Differences between static and dynamic tone mapping are also discussed.

HDR10+ technology has minimum impact for broadcaster workflows and can offer great advantages to the end user, improving image quality and user's experience while viewing video content.

It is royalty and license free technology and is facing a rapid expansion making the technology available to SoCs, receiving display devices vendors and all broadcaster ecosystem (generation, distribution, and reception). This represents a technology easily accessible to adopters, and a growing commitment to delivering a premium HDR experience.

As a developing technology, ST2094-40 can be expanded to technologies in the future, increasing and improving the solutions offered to all workflow, developing tools, compatible displays, and contents catalog, therefore offering more HDR10+ video contents to final user, improving its experience.

HDR10+ Technologies LLC entity was introduced in this paper as responsible to promote the technology and administrates the certification program. As result, several companies have already adopted this technology and, huge gamma of encoding equipments, receiving devices, video codecs, content production, and SW tools support it.

ST 2094-40 metadata offers improved video quality, compared to static metadata tone mapping, as indicated by subjective evaluation tests executed by third part labs.

This paper also listed some advantages and benefits provided by HDR10+ for both: viewers, broadcasters, and equipment vendors.

To conclude, besides other solutions to be adopted for all the components of TV 3.0 architecture taking the DTV system to the next level, the HDR10+ incorporation may offer enhanced feature to broadcast television, enabling the viewer's TV sets to advance seamlessly with the latest technology. HDR10+ is able to deliver the optimal viewing experience across a variety of TV models.

\section{ACKNOWLEDGMENT}

The authors would like to thank SIDIA DTV Lab and Samsung SRA for the support and opportunity. 


\section{REFERENCES}

[1] TV 3.0 Call for Proposal at Forum SBTVD website https://forumsbtvd.org.br/tv3 0/, access on Aug $30^{\text {th }}, 2021$.

[2] T. Ogura and P. Espinosa, "4K HDR Workflow: from Capture to Display," 2018 IEEE Broadcast Symposium (BTS), 2018, pp. 1-9, Oct 2018.

[3] Dynamic Metadata for Color Volume Transform - Application \#4, document SMPTE Standard ST 2094-40:2020, pp.1-29, 16 May 2020.

[4] HDR10+ Technologies website https://hdr10plus.org/; access on Aug $30^{\text {th }}, 2021$.

[5] Vaz, R. A.; Alves, L. G. P.; Akamine, C., "Video Scalability Advantages for the next Brazilian Terrestrial Digital Television Generation (TV 3.0). SET International Journal of Broadcast Engineering, Dec 2020.

[6] Parameter values for ultra-high definition television systems for production and international programme exchange, document ITUR BT.2020-2., 2015.

[7] Image Parameter values for ultra-high definition television systems for use in production and international programme exchange, document ITU-R BT.2100-2., 2018.

[8] High Dynamic Range Electro-Optical Transfer Function of Mastering Reference Displays, document SMPTE Standard ST 2084:2014, pp.1- 14, 29 Aug. 2014

[9] Mastering Display Color Volume Metadata Supporting High Luminance and Wide Color Gamut Images, document SMPT StandardST 2086:2018, pp.1-8, Apr 2018

[10] Ikizyan, Ike, "HDR Dynamic Tone Mapping with Enhanced Rendering Control", SID Symposium Digest of Technical Papers, vol. 50, pp. 303- 306.

[11] A DTV Profile for Uncompressed High Speed Digital Interfaces, document CTA-861-H (2021), Jan 2021.

[12] Web Application Video Ecosystem - Content Specification, document CTA-5001-C, Dec 2020.

[13] HEVC Video Constraints for Cable Television Part 1-1 HDR10 Coding, document ANSI/SCTE 215-1 2020b, 2020

[14] Specification for the use of Video and Audio Coding in Broadcast andBroadband Applications, document DVB BlueBook A001r17, Feb 2021

[15] A/341:2019 Amendment No. 2, ST 2094-40, ATSC A/341 Video HEVC, Sep 2021.

[16] Methodology for The Subjective Assessment of The Quality of Television Pictures, Document Recommendation BT.500, Oct 2019.

[17] Subjective Visual Evaluation of Dynamic HDR metadata system (2094-40) vs static HDR metadata system Verification Tests Report, document BluFocus Quality Assurance Evaluation Report, Nov. 2018.

[18] High Dynamic Range Television for Production and International Programme Exchange, Document Recommendation BT.2390, Apr 2018

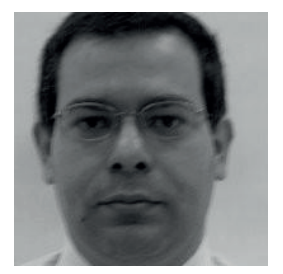

Rodrigo Admir Vaz received the B.S., and M.S. degrees in telecommunications engineering from University of São Paulo (USP), Brazil, in 2003, and 2007, respectively. Currently, he is studying the Ph.D. degree in electrical engineering at Mackenzie Presbyterian University. He has been with SIDIA DTV Lab / SAMSUNG Visual Display (VD) Group since 2007, where he is currently senior engineer. His research interests include next generation terrestrial broadcasting system, High Dynamic Range (HDR), video scalability, broadband and broadcast integration.

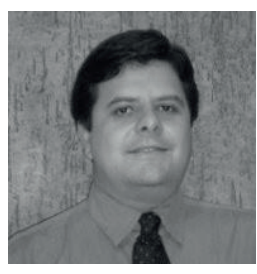

Luiz Gustavo Pacola Alves received his B.Sc. and M.Sc. degrees in Electrical Engineering with emphasis in Computer from University of São Paulo (USP), São Paulo, Brazil, in 2004 and 2008, respectively. He has been working at SIDIA DTV Lab /

SAMSUNG Visual Display (VD) Group since 2009, where he is currently Technical coordinator. He has experience in Standardization \& DTT planning focused on Latin America technical harmonization of Digital TV development in regional standardization bodies. He also has experience in Technical Business Development related to pay TV and live streaming. $\mathrm{He}$ is member of ISDB-T International and Technical Module of the Brazilian Digital Terrestrial Television Forum (SBTVD Forum) with active participation at WGs level representing SAMSUNG, currently engaged on next generation terrestrial broadcasting system with emphasis on High Dynamic Range (HDR).

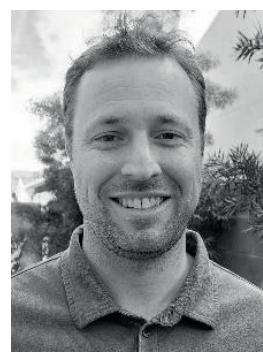

Steve Larson received the B.S. in Electrical and Computer Engineering with an emphasis in Communications Systems from the University of California, San Diego, in 2010. He works as a Technical Program Manager for Samsung Research America (SRA) in the Digital Media Solutions Lab. His areas of focus are high dynamic range (HDR), respective applications to streaming, gaming, live and cinematic content and with the $8 \mathrm{~K}$ video ecosystem working with the $8 \mathrm{~K}$ Association in the Technical Working Group to establish specifications and best practices around $8 \mathrm{~K}$ video formats. 